\title{
M 31's Molecular Arms at All Scales to Below 10 pc
}

\author{
N. Neininger \\ Radioastronomisches Institut der Universität Bonn, Auf dem Hügel 71, \\ D-53121 Bonn, Germany
}

\author{
Ch. Nieten ${ }^{1}$, M. Guélin ${ }^{2}$, H. Ungerechts ${ }^{3}$, R. Lucas ${ }^{2}$, S. Muller ${ }^{2}$, R. \\ Wielebinski ${ }^{1}$
}

${ }^{1}$ MPIfR, D-53121 Bonn (Germany), ${ }^{2}$ IRAM, F-38406 St. Martin

d'Hères (France), ${ }^{3}$ IRAM E-18012 Granada (Spain)

\begin{abstract}
I present a unique data set for the study of molecular gas in galaxies: a complete, high-resolution survey of the CO in M 31 and additional local studies. The fully sampled survey has an angular resolution of $23^{\prime \prime}$ FWHM and the interferometric data attain the pc-scale with subarcsecond resolution. For the first time it is now possible to study large and small scales in conjunction. Thus we are able to derive the global structure and study the links down to the individual cloud complexes and star formation regions.
\end{abstract}

\section{Overview}

We are pursuing a project to study the relationship between molecular clouds and star formation in a single object and for the widest possible range of scales. This needs high sensitivity and complete sampling at high angular resolutions. The best spatial resolution can of course be obtained in our Milky Way, but it is very difficult to investigate the interplay between the various constituents of the interstellar medium (ISM) here. This is due to our "inside view" that makes distance determinations difficult and largely impedes the construction of a global picture of the structure of our Galaxy. The obvious choice for the object thus is a nearby external galaxy and we have started to investigate the molecular content of the closest large spiral: M 31 .

Of course, there have been numerous earlier attempts to derive the distribution of the molecular gas in M 31, but this turned out to be difficult and the first complete survey was published only a few years ago (Dame et al. 1993). Observational techniques new in the field of millimeter-astronomy, such as the On-The-Fly (OTF) method, now allow fully sampled maps of large areas to be obtained in a reasonably short time. Our new map (cf. Fig. 1) clearly shows that the CO emission of M 31 covers only a small percentage of the disk and thus both complete coverage and a well matched angular resolution are necessary.

Once the distribution of the giant molecular cloud complexes (GMCs) was known, we started detailed observations at the highest possible angular resolutions. Several regions were chosen and investigated with the sensitive Plateau 
de Bure interferometer (PdBI). Of particular interest are complexes with peculiarities in their spectra. In contrast to the Galaxy, the kinematical information of the spectra in M 31 can be linked directly to a position. The observed velocity anomalies hence indeed indicate local disturbances.

\section{Observations}

\subsection{The large-scale survey}

The first step for a thorough investigation of the molecular gas content is a complete survey of the entire disk at sufficiently high angular resolution. We used an OTF mapping procedure implemented at the IRAM 30-m telescope to cover an area of about 1 square degree in the ${ }^{12} \mathrm{CO}(1-0)$ transition with a beam of $23^{\prime \prime}$ FWHM. The whole area is covered at least twice, and sampled on a 4 " grid. Several smaller regions of interest have been mapped on a $2^{\prime \prime}$ grid (and correspondingly longer integration times per beam) to obtain high quality data of the (2-1) transition as well. Details about the mapping procedure and the data reduction are given in Neininger (2000).

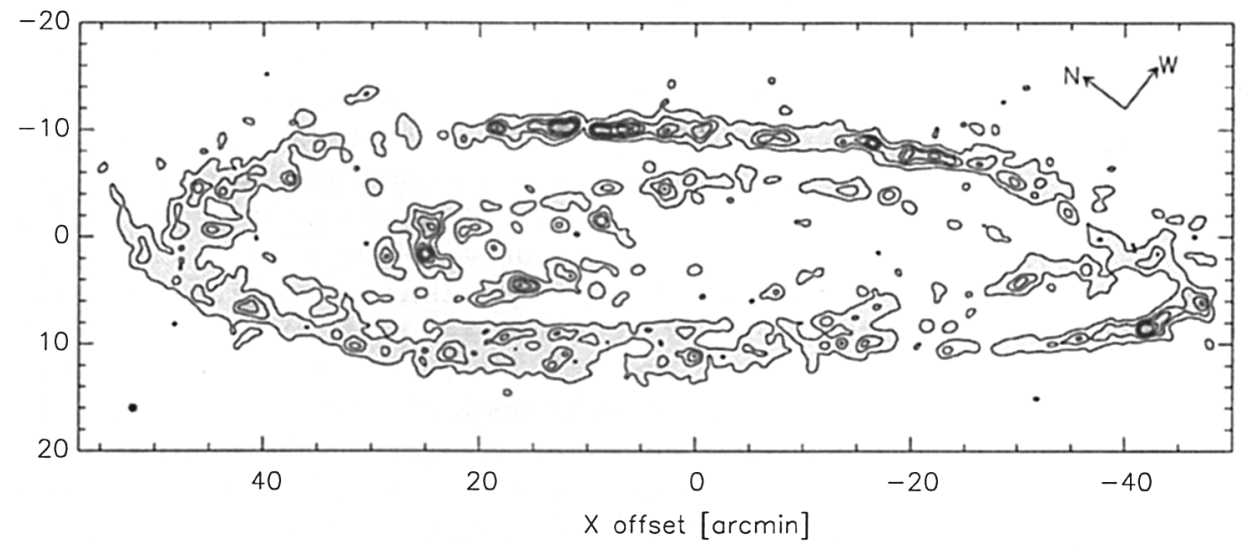

Figure 1. The emission of the ${ }^{12} \mathrm{CO}(1-0)$ in $\mathrm{M} 31$ smoothed to an angular resolution of $45^{\prime \prime} \mathrm{FWHM}$ for clarity (indicated by the dot in the lower left corner). The lowest contour is at a level of at least $4 \sigma$ RMS. The molecular gas is concentrated in thin arms. Nevertheless, a few isolated clouds can be found in the "interarm" regions. Emission has been found between radii of about $4 \mathrm{kpc}$ and $18 \mathrm{kpc}$.

The map (Fig. 1) shows the $\mathrm{CO}$ emission concentrated in narrow arm segments that tend to break up into filamentary pieces. In the western part, we found an arm/interarm contrast of about $10: 1$ for the coherent arm segment (see Nieten et al. 2000) - much higher than that of the atomic gas. No emission is found inside of about $4 \mathrm{kpc}$ radius at the noise level of the survey; close to the nucleus, one cloud complex has been detected at an about 5 times lower intensity, using a special detection strategy (Melchior et al. 2000). 


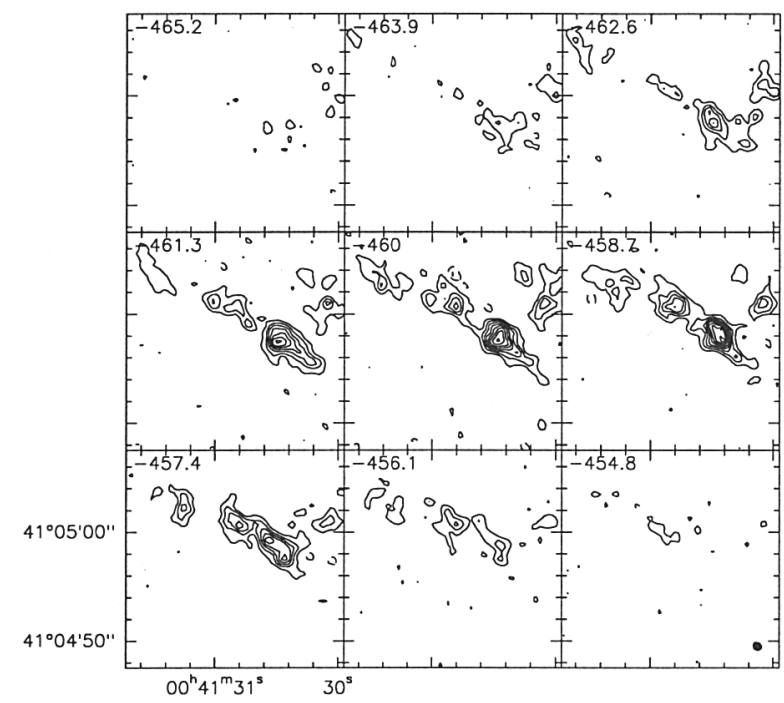

Figure 2. Channel maps of the ${ }^{12} \mathrm{CO}(2-1)$ emission of a GMC at $5 \mathrm{kpc}$ radius. The beam size is $0.9^{\prime \prime} \times 0.74^{\prime \prime}(\simeq 3.5 \mathrm{pc})$, indicated in the lower right corner. Each box is marked with its velocity channel; coordinates are J2000.0. No hint at the obvious substructure is found in the corresponding survey data. Even for this quiescent cloud the virial masses determined from the two data sets differ significantly.

\subsection{Interferometric studies}

For the PdBI observations, we choose GMCs or GMC groups that spread over the area of a few PdBI primary beams while representing a broad variety of the cloud complexes found. The innermost cloud in this sample is located at a radius of $5 \mathrm{kpc}$, the outermost group at $\sim 18 \mathrm{kpc}$. In addition, we choose other GMCs with various morphologies in different environments, including some with multiple spectral components. Up to now, our sample consists of six regions covered with mosaicked observations of 2 to 9 pointings - mainly in the southwestern part of the galaxy, but also containing the prominent association of molecular gas on the northern major axis at a distance of $5 \mathrm{kpc}$ from the center.

Fig. 2 shows as an example the channel maps of a GMC at $5 \mathrm{kpc}$ radius. In the survey it appears as a single entity, with no spatial or kinematical peculiarities. The high-resolution PdBI map shows significant substructure at scales of tens of pc and leads to a different derived virial mass of the complex. Such comparative studies will help to improve mass determinations.

\section{General Results}

On the basis of HI observations (Brinks \& Shane 1984) several kinematical studies have been undertaken to derive the detailed properties of the gaseous disk and spiral arms (e.g. Braun 1991). This is a rather difficult task because of the thickness of the HI disk, its high inclination and the low contrast of the "atomic arms". The survey map of the molecular gas is much better suited to derive the spiral arm structure and to investigate their properties. The small width of the ridges indicates a rather short life time of the molecular gas, but a detailed analysis of the kinematics has still to be done.

There has been a claim of large streaming motions, deduced from early $\mathrm{CO}$ observations at low angular resolution (Ryden \& Stark 1986). Our survey data 
clearly show that the magnitude of non-circular motions in the molecular gas is only of the order of $10 \mathrm{~km} \mathrm{~s}^{-1}$ which is also the typical line width. A comparison with the atomic gas shows that the $\mathrm{CO}$ is situated in the middle of the "atomic arms", where the velocity gradient vanishes (Nieten et al., in prep.). The total range of the widths of individual spectral components is about 4 to $15 \mathrm{~km} \mathrm{~s}^{-1}$. However, at many places we found strong small-scale disturbances.

Typical examples are double- or multiple-component spectra, broad lines and short-range spatial variations. From the observational geometry we are led to conclude that we are not observing chance line-of-sight coincidences of spatially separated clouds, but true local effects. Separation of velocity components reach $50 \mathrm{~km} \mathrm{~s}^{-1}$, much more than the intrinsic line width. The sizes of such regions are of the order of a few primary beams of the PdBI, hence ideally suited for a detailed observation.

A statistical analysis of the GMCs as well as a more detailed investigation of the properties of the individual clumps is under way. Already the few cloud complexes investigated thus far hint however at important implications for the properties of molecular gas agglomerations and their investigation:

* CO spectra with multiple components are usually confined to small regions.

* The observational geometry indicates a true local phenomenon.

* The substructure of such GMCs consists of well separated filaments or clumps.

* The velocity separation of the components may be significantly greater than the streaming motions or the line width.

* Even seemingly quiescent clouds may consist of individual clumps which may afflict the derivation of mass or temperature.

This is the first time that such a wide range of scales of the molecular gas emission can be investigated and searched for clues about its state, evolution into stars and mutual interactions with the other constituents of the ISM.

Acknowledgments. The IRAM technical staff provided excellent receivers, hard- and software for the new observing modes used. In particular, it is a pleasure to thank the PdBI staff and astronomers for the superb observations.

\section{References}

Braun, R. 1991, A\&A 372, 54

Brinks, E., Shane, W.W. 1984, A\&AS 55, 179

Dame, T.M., Koper, E., Israel, F.P., Thaddeus, P. 1993, ApJ 418, 730

Melchior, A.-L., et al. 2000, MNRAS 312, L29

Neininger, N. 2000, in ASP Conf. Ser. Vol. 217 Imaging at Radio through Submillimeter Wavelengths, ed. J. Mangum (San Francisco: PASP), 72

Neininger N. 1999, in The Physics and Chemistry of the Interstellar Medium, ed. V. Ossenkopf et al. (Herdecke: GCA), 42

Neininger, N., et al. 1998, Nat., 395, 871

Nieten, Ch., et al. 2000, in Proceedings of the 232. WE-Heraeus-Seminar, ed. E. Berkhuijsen et al. (Aachen: Shaker), 21

Ryden, B.S., Stark, A.A. 1986, ApJ 305, 823 